\title{
INTERVALO HÍDRICO ÓTIMO NUM NITOSSOLO VERMELHO DISTROFÉRRICO IRRIGADO ${ }^{(1)}$
}

\author{
Everton Blainski ${ }^{(2)}$, Antonio Carlos Andrade Gonçalves ${ }^{(3)}$, Cássio Antonio \\ Tormena $^{(3)}$, Marcos Vinicius Folegatti ${ }^{(4)}$ \& Rachel Muylaert Locks \\ Guimarães $^{(2)}$
}

\begin{abstract}
RESUMO
O manejo da irrigação tem-se baseado no controle do potencial da água no solo ( $\Psi)$ como fator limitante do crescimento das plantas. Entretanto, outras variáveis podem influenciar a cultura mesmo que o $\Psi$ não seja limitante. O Intervalo Hídrico Ótimo (IHO) é um conceito de disponibilidade de água no solo que leva em consideração a porosidade de aeração e a resistência do solo à penetração em adição ao $\Psi$. $O$ objetivo deste estudo foi quantificar o IHO num Nitossolo Vermelho distroférrico irrigado e utilizá-lo no estabelecimento de critérios para o manejo de água e do solo em áreas irrigadas. A resistência do solo à penetração foi a variável que limitou o IHO com maior frequência, diminuindo sua magnitude com o aumento da densidade do solo (Ds). Com o aumento da Ds, ocorreu redução na frequência com que $\theta$ manteve-se dentro dos limites do IHO. A Ds crítica (Dsc) foi de $1,40 \mathrm{Mg} \mathrm{m}^{-3}$, indicando severa degradação física do solo para Ds $>$ Dsc. Para Ds $<1,28 \mathrm{Mg} \mathrm{m}^{-3}$, o $\Psi$ de $-800 \mathrm{hPa}$ utilizado frequentemente para o controle da irrigação por meio de tensiômetros caracterizou o limite inferior do IHO. Para $1,28<\mathrm{Ds} \leq 1,40 \mathrm{Mg} \mathrm{m}^{-3}$, o limite inferior do IHO foi determinado pela RP e, nestas condições, a adoção de $\Psi$ igual a -800 hPa como limite para a aplicação de água estabelece restrições físicas às plantas devido à elevada $R P$. Neste caso, o mapeamento de áreas com $D s$ uniformes poderia ser utilizado para o manejo do $\Psi$ para a manutenção da $R P<2,0 \mathrm{MPa}$. Nas áreas em que a $\mathrm{Ds}<1,28 \mathrm{Mg} \mathrm{m}^{-3}$ pode ocorrer maior secamento do solo sem que ocorra $R P>2,0 \mathrm{MPa}$; para $1,28<\mathrm{Ds} \leq 1,40 \mathrm{Mg} \mathrm{m}^{-3}$ deve-se manter $\Psi>-800 \mathrm{hPa}$ visando ao controle da $\mathrm{RP}$. Para áreas em que Ds > Dsc, medidas que visem a redução da Ds poderiam ser tomadas em função da severa degradação física do solo.
\end{abstract}

Termos de indexação: irrigação, qualidade física do solo, resistência do solo à penetração.

\footnotetext{
(1) Parte da Dissertação de Mestrado do primeiro autor apresentada ao Programa de Pós-Graduação em Agronomia da Universidade Estadual de Maringá - UEM. Recebido para publicação em março de 2008 e aprovado em janeiro de 2009.

(2) Doutorando do Programa de Pós-Graduação em Agronomia da Universidade Estadual de Maringá - PGA/UEM. Av. Colombo 5790, CEP 87020-900 Maringá (PR). E-mail: evertonblainski@gmail.com; rachellocks@gmail.com

(3) Professor Adjunto do Departamento de Agronomia, UEM. Bolsista do CNPq. E-mails: acagoncalves@uem.br; catormena@uem.br

(4) Professor Titular do Departamento de Engenharia Rural da Escola Superior de "Agricultura Luiz de Queiroz" - ESALQ/USP. Caixa Postal 9, Av. Pádua Dias 11, CEP 13418-400 Piracicaba (SP). E-mail: mvfolega@esalq.usp.br
} 


\title{
SUMMARY: LEAST LIMITING WATER RANGE OF AN IRRIGATED DYSTROFERRIC RED NITOSOL
}

\begin{abstract}
The establishment of irrigation management has been based on the soil water potential $(\Psi)$ as a limiting factor for plant growth. However, other variables can affect crop growth even when $\Psi$ is not limiting. The least limiting water range $(L L W R)$ is a concept of available water that take account the influence of aeration and soil resistance to penetration (SR) in addition to $\Psi$. The objective of this study was to quantify the LLWR in an irrigated Dystroferric Red Nitosol and to use it to determine the soil and water management for irrigated areas. Soil penetration resistance limited LLRW most often, reducing its magnitude with the increase of soil bulk density $(B d)$. Therefore, the higher $B d$, the less often $\theta$ was inside the limits of $L L W R$. The critical Bd (Bdc) was $1.40 \mathrm{Mg} \mathrm{m}^{-3}$, indicating severe soil physical degradation when $B d>B d c$. For $B d<1.28 \mathrm{Mg} \mathrm{m}^{-3}$, the $\Psi=-800 \mathrm{hPa}$, which is frequently used for irrigation management using tensiometers, characterized the lower limit for LLWR. For $1.28<B d<$ $1.40 \mathrm{Mg} \mathrm{m}^{-3}$ the lower limit was determined by $S R$, and under these conditions $\Psi=-800 \mathrm{hPa}$ as a limit for water application results in physical restrictions for plants due to the high SR. In this case, the mapping of areas with homogenous Bd could be used for the management of $\Psi$ to maintain $S R<2.0 \mathrm{MPa}$. In areas where $B d<1.28 \mathrm{Mg} \mathrm{m}^{-3}$ soil drying can be higher without $S R>2.0 \mathrm{MPa}$; for areas in which $1.28<B d<1.40 \mathrm{Mg} \mathrm{m}^{-3} \Psi$ should be maintained at $>$. 800 hPa to maintain adequate SR. For areas where Bd $>B d$ c measures to reduce Bd could be taken due to the severe soil physical degradation in this situation.
\end{abstract}

Index terms: irrigation, soil physical quality, soil resistance to penetration.

\section{INTRODUÇÃO}

O manejo da água, em sistemas irrigados deve ser efetuado de forma a proporcionar o controle do potencial da água no solo $(\Psi)$, permitindo às culturas expressarem o seu potencial genético de produtividade. A definição do momento de irrigar deve ser baseada em técnicas que determinem em que condição as plantas sofrem restrições. O controle do $\Psi$ é o método mais difundido e de maior simplicidade para a manutenção do teor de água no solo $(\theta)$ dentro de uma faixa adequada para o crescimento e desenvolvimento das plantas (Campbell, 1988; Kramer \& Boyer, 1995).

A aplicação de água, além de estabelecer modificações no $\theta$ e no $\Psi$, acarreta alterações em outras propriedades físicas do solo, como a porosidade de aeração (PA) e a resistência do solo à penetração (RP), influenciando processos fisiológicos das plantas (Letey, 1985). Em solos bem estruturados, a faixa de $\theta$ em que as condições físicas do solo são, teoricamente, adequadas às plantas, é definida pelo intervalo compreendido entre a capacidade de campo $\left(\theta_{\mathrm{CC}}\right)$ e o ponto de murcha permanente $\left(\theta_{\mathrm{PMP}}\right)$, reconhecida como de água disponível (AD) (Reichardt \& Timm, 2004; Libardi, 2005). Entretanto, a degradação física do solo evidenciada pelo aumento na densidade do solo (Ds) resulta em modificações no sistema poroso com efeitos danosos às plantas. Isso decorre da redução da porosidade total do solo a expensas da macroporosidade, reduzindo as trocas de gases entre o solo e a atmosfera e aumentando a RP, o que poderia atuar como fator limitante ao crescimento das plantas dentro da faixa de AD (Letey, 1985; Topp et al., 1994).
O Intervalo Hídrico Ótimo (IHO) define uma faixa de $\theta$ em que são mínimas as restrições físicas ao crescimento das plantas, representando um avanço em relação à $\mathrm{AD}$ por incluir as limitações devidas à RP e PA em adição ao efeito do $\Psi$ (Silva et al., 1994; Tormena et al., 2007). A maior parte dos resultados de pesquisa mostra que o IHO reduz com a compactação do solo, isto é, com o aumento da Ds (Silva \& Kay, 1997; Tormena et al., 1999; Imhoff et al., 2001, Cavalieri et al., 2006). A Ds em que o IHO $=0$ é definida como densidade do solo crítica (Dsc), e quando Ds > Dsc, há indicações de severa degradação estrutural do solo. Assim, sistemas de manejo que proporcionem redução no IHO expõem as culturas a maior frequência de estresses por excessiva RP ou reduzida PA (Silva \& Kay, 1997; Klein \& Camara, 2007) em função de variações temporal e espacial de $\theta$, em condições naturais ou sob irrigação.

Dentre as propriedades físicas do solo diretamente ligadas ao crescimento das plantas, a RP é a que mais frequentemente reduz o IHO (Tormena et al., 1999, 2007; Zou et al., 2000; Imhoff et al., 2001; Beutler et al., 2007). Valores de RP entre 1,0 e 3,5 MPa podem restringir o crescimento e desenvolvimento das raízes (Benghough \& Mullins, 1990). No entanto, para culturas anuais, o valor de $\mathrm{RP}=2 \mathrm{MPa}$ tem sido largamente empregado na quantificação do IHO (Silva et al., 1994, Tormena et al., 1999; Araujo et al., 2004). Em solos compactados, com solo úmido a PA pode restringir o IHO (Lapen et al., 2004), prejudicando o crescimento e desenvolvimento vegetal pela reduzida difusão de $\mathrm{O}_{2}$. Por outro lado, um rápido aumento de $\mathrm{RP}$ com o secamento do solo pode se tornar um fator impeditivo ao crescimento das plantas (Blainski et al., 2008). 
De acordo com Kay (1990), quanto menor o IHO maior será a probabilidade de ocorrência de estresses físicos pela reduzida difusão de gases no solo ou pela elevada RP. Silva \& Kay (1997) associaram medidas temporais de $\theta$ com os limites do IHO para quantificar a frequência com que $\theta$ ocorre fora dos limites do IHO ou, alternativamente, a frequência com que $\theta$ ocorre dentro do IHO $\left(F_{\text {dentro }}\right)$. Eles demonstraram que há uma relação direta entre a magnitude do $\mathrm{IHO}$ e $F_{\text {dentro }}$. Neste sentido, Benghough et al. (2006) ressaltam que o IHO em associação com as variações espaciais e temporais de $\theta$ é a melhor estratégia para indicar as possíveis restrições físicas às plantas, sugerindo que a variação de $\theta$ pode ser utilizada para a obtenção de condições físicas favoráveis ao crescimento e desenvolvimento das plantas. Portanto, o IHO pode ser utilizado para definir estratégias de aplicação e manejo de água em áreas irrigadas. Além disso, por meio do IHO pode-se estimar a Dsc e utilizá-la como critério para o controle e monitoramento da qualidade física do solo.

Os objetivos deste estudo foram quantificar o IHO num Nitossolo Vermelho distroférrico irrigado e propor sua utilização como critério para o manejo de água e do solo em áreas irrigadas e para o monitoramento da qualidade física do solo.

\section{MATERIAL E MÉTODOS}

O trabalho foi realizado em área experimental da Universidade Estadual de Maringá, localizada no município de Maringá-PR (23 25'S $\left.51^{\circ} 57^{\circ} \mathrm{W}\right)$, com altitude média de $540 \mathrm{~m}$. O clima da região é caracterizado como Cfa, com precipitação pluvial média anual de $1.500 \mathrm{~mm}$ (Iapar, 2000). O solo da área é classificado como Nitossolo Vermelho distroférrico (Embrapa, 2006). A análise granulométrica na camada de 0-0,20 m, realizada conforme Embrapa (1997), indicou valores médios de $175 \mathrm{~g} \mathrm{~kg}^{-1}$ de areia, 90 de silte e 735 de argila.

O experimento foi realizado em área sob irrigação por aspersão, com aspersores espaçados em $15 \times 18 \mathrm{~m}$, com diâmetro de bocais de 5,6 x 3,2 mm. O preparo do solo foi realizado por meio de um escarificador com cinco hastes estreitas espaçadas de $0,30 \mathrm{~m}$ a uma profundidade média de $0,25 \mathrm{~m}$, seguido de uma gradagem niveladora. A cultura do feijoeiro (Phaseolus vulgaris L. cv. IAPAR 81) foi implantada no dia 12 de abril de 2006, utilizando-se 15 sementes por metro e espaçamento entre linhas de $0,50 \mathrm{~m}$ (população de 300.000 plantas ha $^{-1}$ ). A adubação foi feita de acordo com a análise de solo, e os demais tratos culturais foram realizados conforme recomendações para a cultura no Estado do Paraná (Iapar, 1989).

Após o plantio, a área experimental foi divida em oitenta parcelas experimentais com dimensões de 3 × $3 \mathrm{~m}\left(9 \mathrm{~m}^{2}\right)$. No centro de cada parcela experimental, foi instalada verticalmente uma sonda de TDR
(Time Domain Reflectometry) com 0,20 m de comprimento para o monitoramento do $\theta$. O monitoramento diário do $\theta$ da camada $0-0,20 \mathrm{~m}$ iniciou-se na fase reprodutiva da cultura e estendeu-se até a maturação fisiológica, ou seja, entre os dias 131 e 191 do corrente ano.

Após a colheita, realizada no dia 10 de agosto de 2006, procedeu-se à amostragem do solo no centro de cada parcela, na porção intermediária da camada de 0-0,20 m, utilizando anéis metálicos (diâmetro e altura de $0,05 \mathrm{~m})$, totalizando 80 amostras de solo indeformadas. Imediatamente após a retirada das amostras, elas foram envolvidas em papel-alumínio e acondicionadas em saco-plástico até serem preparadas para análise. No laboratório, as amostras foram saturadas mantendo-se uma lâmina de água de até dois terços da altura dos anéis. Para a determinação da curva de retenção de água no solo (CRA) e da curva de resistência do solo à penetração (CRS), as amostras foram divididas em dez grupos de oito amostras, e cada grupo foi sorteado para um dos seguintes potenciais: - $10,-20,-40,-60,-80$ e $-100 \mathrm{hPa}(\mathrm{hPa}$ é igual a $\mathrm{cm}$ de coluna de água), estabelecidos por meio da diferença de nível em uma coluna de água com uma mesa de tensão (Romano et al., 2002) e aos potenciais de $-500,-1.000,-8.000$ e $-15.000 \mathrm{hPa}$ aplicados por meio de pressões em placas porosas conforme Dane \& Hopmans (2002). Após atingirem o equilíbrio em cada potencial, as amostras foram pesadas e, em seguida, foi determinada a RP utilizando um penetrômetro eletrônico descrito por Tormena et al. (1999). Em seguida, as amostras foram secas em estufa a $\pm 105^{\circ} \mathrm{C}$, por $24 \mathrm{~h}$, para a determinação de $\theta$ e de Ds (Blake \& Hartge, 1986).

A CRA foi expressa pela relação entre $\theta$ e $\Psi$ conforme Ross et al. (1991). A Ds foi incorporada ao modelo, seguindo o procedimento descrito por Leão et al. (2005), conforme equação 1:

$$
\theta=a \Psi^{b} \mathrm{Ds}^{c}
$$

em que $\theta$ : teor de água no solo $\left(\mathrm{m}^{3} \mathrm{~m}^{-3}\right) ; \Psi$ : potencial da água no solo (hPa); Ds: densidade do solo ( $\left.\mathrm{Mg} \mathrm{m}^{-3}\right)$, e $a, b, c$ são os coeficientes obtidos no ajuste da equação 1 aos dados.

Os dados de RP foram ajustados em função de $\theta$ e da Ds utilizando-se a função proposta por Busscher (1990), descrita na equação 2:

$$
\mathrm{RP}=d \theta^{e} \mathrm{Ds}^{f}
$$

em que RP: resistência do solo à penetração (MPa), e $d, e, f$ são os coeficientes obtidos no ajuste da equação 2 aos dados.

Os ajustes das equações 1 e 2 foram feitos pela rotina PROC NLIN empregando o programa estatístico SAS (SAS, 1999).

O IHO foi determinado conforme Silva et al. (1994). Os limites superior e inferior de $\Psi$ de $-60 \mathrm{hPa}$ e de $-15.000 \mathrm{hPa}$ definiram $\theta$, respectivamente, na capacidade de campo - $\theta_{\mathrm{CC}}$ (Reichardt, 1988) e no ponto 
de murcha permanente - $\theta_{\text {PMP }}$ (Savage et al., 1996), ambos obtidos a partir da equação 1 . A capacidade de campo, aceita como limite superior do IHO, deve ser considerada um conceito arbitrário por ser resultado de um comportamento dinâmico da água no solo e não uma característica intrínseca da matriz do solo (Reichardt, 1988). Com isso, o $\Psi$ associado a $\theta_{\mathrm{CC}}$ pode variar com características do perfil e do volume de drenagem aceito como desprezível. O $\Psi$ de $-60 \mathrm{hPa}$ adotado como potencial correspondente ao $\theta_{\mathrm{CC}}$ foi previamente determinado por meio do método do perfil instantâneo, seguindo procedimento descrito por Libardi (2005).

Em cada amostra foi calculado um valor de $\theta$ em que a porosidade de aeração é de $10 \%$ - $\theta_{\mathrm{PA}}$ (Grable \& Siemer, 1968) utilizando-se a expressão [(1-Ds/Dp)$0,1]$, sendo Dp a densidade média de partículas. Foi utilizado um valor médio de $\mathrm{Dp}=3,17 \mathrm{Mg} \mathrm{m}^{-3}$, determinado previamente para o solo da área experimental conforme Embrapa (1997). Para a determinação de $\theta$ em que a RP atinge o valor considerado crítico de $2 \mathrm{MPa}\left(\theta_{\mathrm{RP}}\right)$, foi utilizada a equação 2 reescrita conforme a equação 3 :

$$
\theta_{\mathrm{RP}}=\left[\left(\frac{\mathrm{RP}}{d \cdot \mathrm{Ds}^{f}}\right)^{\frac{1}{e}}\right]
$$

O IHO foi determinado considerando como o limite superior o menor valor entre $\theta_{\mathrm{CC}}$ e $\theta_{\mathrm{PA}}$ e como limite inferior o maior valor entre $\theta_{\mathrm{PMP}}$ e $\theta_{\mathrm{RP}}$.

A partir da determinação do IHO e das medidas de $\theta$, foi determinada a frequência com que $\theta$ manteve-se dentro dos limites do IHO ( $\left.F_{\text {dentro }}\right)$. A $F_{\text {dentro }}$ foi determinada para os locais que apresentaram as seguintes condições físicas: máximo $\mathrm{IHO}\left(\mathrm{IHO}_{\mathrm{Max}}\right)$, IHO correspondente a 80, 70, 50 e $25 \%$ do IHO $_{\text {Max }}$ $\left(\mathrm{IHO}_{80 \%} ; \mathrm{IHO}_{70 \%} ; \mathrm{IHO}_{50 \%} \mathrm{e} \mathrm{IHO}_{25 \%}\right.$ respectivamente) $\mathrm{e}$, ainda, $\mathrm{IHO}=0\left(\mathrm{IHO}_{\text {nulo }}\right)$.

\section{RESULTADOS E DISCUSSÃO}

Os momentos estatísticos para as variáveis Ds, $\theta$ e RP são apresentados no quadro 1. A variável RP apresentou coeficiente de variação (CV) maior que as demais variáveis, por causa das medidas terem sido realizadas numa ampla variação de $\theta$ e Ds. Resultados semelhantes foram obtidos por Silva et al. (1994), Tormena et al. (1998, 1999), Imhoff et al. (2001) e Tormena et al. (2007).

As equações 1 e 2 explicaram $95 \%$ da variabilidade de $\theta$ e $91 \%$ da variabilidade de RP, respectivamente (Quadro 2). O intervalo de confiança dos coeficientes não inclui o valor igual a zero, possibilitando afirmar que os modelos são significativos ( $\mathrm{p}>\mathrm{F}=0,001)$. Os resíduos apresentaram distribuição normal pelo teste de Shapiro-Wilk, indicando que se ajustaram aos dados adequadamente. A RP foi positivamente correlacionada com Ds e negativamente correlacionada com $\theta$, também verificado por Silva et al. (1994), Tormena et al. (1999) e Imhoff et al. (2001). O aumento da RP com o decréscimo de $\theta$ pode ser associado à maior coesão entre as partículas minerais particularmente em solos de textura argilosa (Kay \& Angers, 1999). Com o incremento da Ds, tanto a maior fricção entre as partículas quanto a maior coesão atuam como os principais mecanismos para o aumento da RP (Vepraskas, 1984). A influência positiva da Ds sobre a CRA pode ser atribuída à redistribuição do tamanho de poros com aumento na proporção de poros de menor diâmetro, de modo a se tornarem mais eficientes na retenção de água em elevados $\Psi$. Em baixos $\Psi$, a influência da Ds sobre a CRA pode ser atribuída à maior massa de sólidos com elevada superfície de adsorção, uma vez que a compactação geralmente não altera a microporosidade dentro dos agregados. Esses resultados estão de acordo com os resultados obtidos por Silva et al. (1994); Tormena et al. (1998, 1999); Imhoff et al. (2001); e Leão et al. (2004).

O intervalo hídrico ótimo (IHO) está representado pela área hachurada da figura 1 e apresentou uma relação negativa com a Ds em concordância com os resultados obtidos por Silva et al. (1994), Leão et al. (2005) e Cavalieri et al. (2006). O limite superior do IHO é determinado por $\theta_{\mathrm{CC}}$ para $\mathrm{Ds}<1,35 \mathrm{Mg} \mathrm{m}^{-3}$, a partir desta Ds o limite superior do IHO passa a ser determinado por $\theta_{\mathrm{PA}}$ (Figura 1). O limite inferior do IHO foi determinado por $\theta_{\mathrm{RP}}$ em toda a extensão de Ds. Resultados similares foram verificados por Imhoff et al. (2001), Klein \& Camara (2007) e Tormena et al.

Quadro 1. Momentos estatísticos das variáveis físicas do solo determinadas nas amostras indeformadas coletadas na área experimental

\begin{tabular}{cccccc}
\hline Variável & Mínimo & Média & Máximo & Desvio-padrão & CV \\
\hline & & & & & $\%$ \\
$\operatorname{Ds~}\left(\mathrm{Mg} \mathrm{m}^{-3}\right)$ & 1,11 & 1,36 & 1,47 & 0,0694 & 5,11 \\
$\theta \quad\left(\mathrm{m}^{3} \mathrm{~m}^{-3}\right)$ & 0,28 & 0,44 & 0,53 & 0,0643 & 14,68 \\
$\mathrm{RP}(\mathrm{MPa})$ & 0,29 & 3,16 & 12,66 & 3,2920 & 104,3 \\
\hline
\end{tabular}

$\mathrm{N}=80$, Ds: densidade do solo $\left(\mathrm{Mg} \mathrm{m}^{-3}\right)$, $\theta$ : conteúdo de água $\left(\mathrm{m}^{3} \mathrm{~m}^{-3}\right)$; RP: resistência do solo à penetração $(\mathrm{MPa})$. 
Quadro 2. Estimativas dos coeficientes de regressão para a curva de retenção de água no solo $\left(\psi=a \emptyset^{b} D s^{c}\right)$ e para a curva de resistência do solo à penetração $\left(\mathrm{RP}=d \theta^{e} \mathrm{Ds} \mathrm{s}^{f}\right)$. $\theta$ é conteúdo de água no $\mathrm{solo}\left(\mathrm{m}^{3} \mathrm{~m}^{-3}\right)$, $\psi$ é o potencial de água no solo $(\mathrm{hPa})$, Ds é a densidade do solo $\left(\mathrm{Mg} \mathrm{m}^{-3}\right)$ e $\mathrm{RP}$ é a resistência do solo à penetração $(\mathrm{MPa})$

\begin{tabular}{|c|c|c|c|c|}
\hline \multirow{2}{*}{ Parâmetro } & \multirow{2}{*}{ Valor estimado } & \multirow{2}{*}{ Erro-padrão } & \multicolumn{2}{|c|}{ Intervalo de confiança } \\
\hline & & & Limite inferior & Limite superior \\
\hline \multicolumn{5}{|c|}{ Curva de retenção de água no solo $\left(p>F=0,001 ; R^{2}=0,95\right)$} \\
\hline$a$ & 0,5343 & 0,0052 & 0,5239 & 0,5451 \\
\hline$b$ & $-0,0565$ & 0,0005 & $-0,0575$ & $-0,0556$ \\
\hline$c$ & 0,2997 & 0,0341 & 0,2401 & 0,3594 \\
\hline \multicolumn{5}{|c|}{ Curva de resistência do solo à penetração $\left(p>F=0,001 ; R^{2}=0,91\right)$} \\
\hline$d$ & 0,0013 & 0,0006 & 0,0003 & 0,0023 \\
\hline$e$ & $-5,7425$ & 0,2963 & $-6,2374$ & $-5,2477$ \\
\hline$f$ & 8,5011 & 0,7409 & 7,2636 & 9,7385 \\
\hline
\end{tabular}

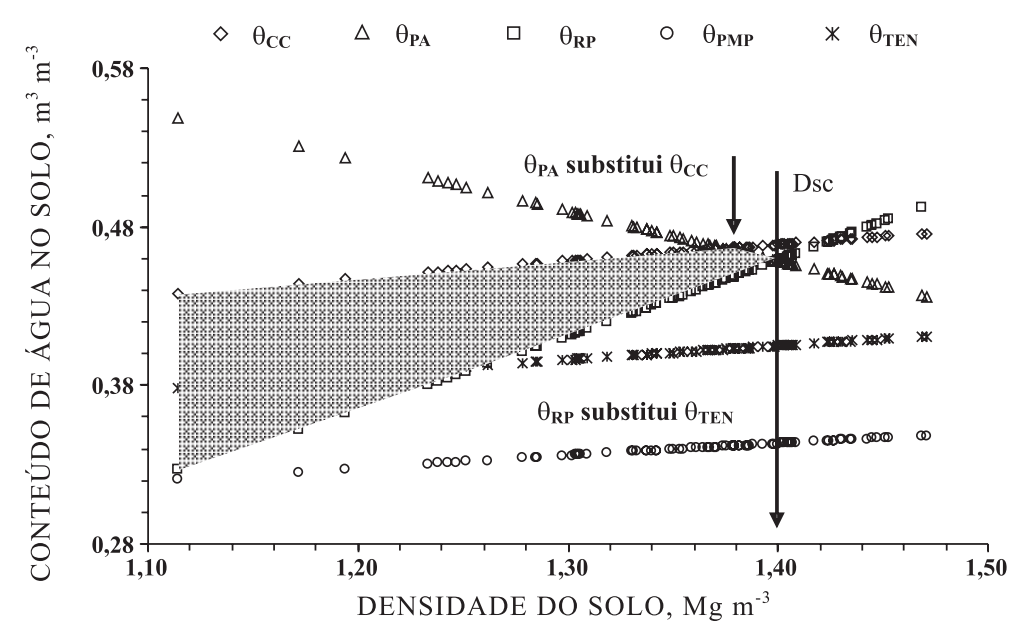

Figura 1. Variação do conteúdo de água com a densidade do solo em relação à capacidade de campo $\left(\theta_{\mathrm{CC}}\right)$, à porosidade de aeração $\left(\theta_{\mathrm{PA}}\right)$, à resistência do solo à penetração $\left(\theta_{\mathrm{RP}}\right)$, ao ponto de murcha permanente $\left(\theta_{\mathrm{PMP}}\right)$ e ao potencial de $-800 \mathrm{hPa}\left(\theta_{\mathrm{TEN}}\right)$ adotado para controle de irrigação.

(2007), apesar das diferenças nos valores de Ds devido às distintas composições granulométricas e mineralógicas dos solos. A RP foi a variável que mais frequentemente reduziu o IHO com o aumento da Ds, como pode ser verificado em Silva et al. (1994) e Tormena et al. (1999). Portanto, estratégias que possibilitem a redução da RP poderiam ser adotadas a fim de aumentar o IHO. A redução de RP pode ocorrer mediante práticas mecânicas para diminuir a Ds ou pelo manejo da irrigação, mantendo-se $\theta$ acima do nível crítico determinado $\left(\theta_{\mathrm{RP}}\right)$, em concordância com os resultados obtidos por Imhoff et al. (2001). Todavia, sistemas de manejo como o plantio direto associado à rotação de culturas, propiciam melhorias na qualidade física do solo e o surgimento de bioporos estáveis, que serviriam como rotas alternativas para o crescimento das raízes quando $\mathrm{RP}>2,0 \mathrm{MPa}$
(Williams \& Weil, 2004). Ehlers et al. (1983) relatam que é possível o crescimento prolífico de raízes com $\mathrm{RP}>3,5 \mathrm{MPa}$ associado à presença dos bioporos. Em solos tropicais, $\mathrm{RP}=3,5 \mathrm{MPa}$ tem sido utilizado como crítico na quantificação do IHO em sistema de plantio direto (Tormena et al., 2007). Além disso, o incremento da matéria orgânica poderia reduzir a sensibilidade do solo e aumentar a RP devido ao aumento da Ds ou ao secamento do solo (Kay et al., 1997).

A densidade do solo crítica (Dsc) é de 1,40 $\mathrm{Mg} \mathrm{m}^{-3}$ (Figura 1). A ocorrência de Ds $>$ Dsc sinaliza para a degradação física do solo (Silva et al., 1994). Para Ds > Dsc, as condições físicas do solo são altamente restritivas independentemente de $\theta$, pela reduzida aeração ou pela excessiva resistência do solo à penetração. Nessas condições, técnicas de manejo que 
proporcionem a redução da Ds devem ser empregadas visando à melhoria da qualidade física do solo. A escarificação do solo promove a redução da Ds e da RP com o mínimo possível de movimentação do solo, apesar do efeito temporário desta prática (Busscher et al., 1995, 2002).

A variação temporal de $\theta$ em relação ao IHO para diferentes Ds é apresentada na figura 2. Os resultados são compatíveis com os de Silva \& Kay (1997) e Klein \& Câmara (2007), que mostram que, com o aumento da Ds ou redução do IHO, ocorre maior proporção de $\theta$ fora dos limites do IHO, como foi sugerido por Kay (1990). O limite superior do IHO pouco influenciou na $F_{\text {dentro }}$, mesmo com elevadas Ds. Nos dias 138 e 180 foram aplicadas as maiores lâminas de irrigação, e $\theta$ medidos imediatamente após as aplicações de água apresentaram valores acima do limite superior do IHO (Figura 2). No entanto, poucas horas depois, $\theta$ atingiu valores menores que o limite superior do IHO, indicando mínimas restrições de aeração neste solo. Com a diminuição do IHO, ocorre menor $F_{\text {dentro }}$, ou seja, aumenta a probabilidade das plantas sofrerem estresse por elevada RP. Para Ds $=1,11 \mathrm{Mg} \mathrm{m}^{-3}$ $\left(\mathrm{IHO}_{\mathrm{Max}}\right), \theta$ mantiveram-se praticamente todo o tempo dentro dos limites do IHO $\left(F_{\text {dentro }}=98 \%\right)$. Com o aumento da Ds e a consequente diminuição do IHO, a
$F_{\text {dentro }}$ atinge $80 \%$ para $\mathrm{Ds}=1,19 \mathrm{Mg} \mathrm{m}^{-3}\left(\mathrm{IHO}_{80 \%}\right)$. Para Ds $=1,23 ; 1,30 ;$ e $1,36 \mathrm{Mg} \mathrm{m}^{-3}$, a $F_{\text {dentro }}$ reduz para $50 ; 17$; e $2 \%$ respectivamente $\left(\mathrm{IHO}_{70} \%, \mathrm{IHO}_{50} \%\right.$ e $\left.\mathrm{IHO}_{25} \%\right)$. Na condição em que o IHO = $0\left(\mathrm{IHO}_{\text {nulo }}\right)$, ou seja, Ds $=$ Dsc $\left(1,40 \mathrm{Mg} \mathrm{m}^{-3}\right)$, as condições físicas do solo são extremamente deletérias às plantas.

Uma estratégia para aumentar a $F_{\text {dentro }}$ pode ser fundamentada na utilização de tensiômetros para o controle do $\Psi$ em sistemas irrigados (Lopes et al., 2004), com o limite inferior de $\theta$ associado ao $\Psi=$ $-800 \mathrm{hPa}\left(\theta_{\mathrm{TEN}}\right)$. Entretanto, os resultados mostram que, para $\mathrm{Ds} \geq 1,28 \mathrm{Mg} \mathrm{m}^{-3}$, o $\theta_{\mathrm{RP}}$ substitui o $\theta_{\mathrm{TEN}}$ como limite inferior do IHO. Essa condição ocorre em cerca de $88 \%$ das amostras coletadas, ou seja, a adoção do $\Psi=-800 \mathrm{hPa}$ como limite para manejo de irrigação expõe a cultura a $\mathrm{RP}>2,0 \mathrm{MPa}$ em $88 \%$ da área irrigada. Isso sugere que a utilização de um $\Psi$ fixo para o manejo da irrigação pode prejudicar o desenvolvimento da cultura em função da variabilidade espacial das propriedades físicas do solo.

A estreita relação entre $\Psi, \theta, D$ s e RP permite que a partir de $\theta_{\mathrm{RP}}$ obtido por meio da curva de resistência do solo à penetração (Equação 3) seja estimado o $\Psi$ em que a RP atinge o valor crítico estabelecido $\left(\Psi_{\mathrm{RP}}\right)$ por meio da equação 4 , reescrita a partir da curva de retenção de água no solo (Equação 1):

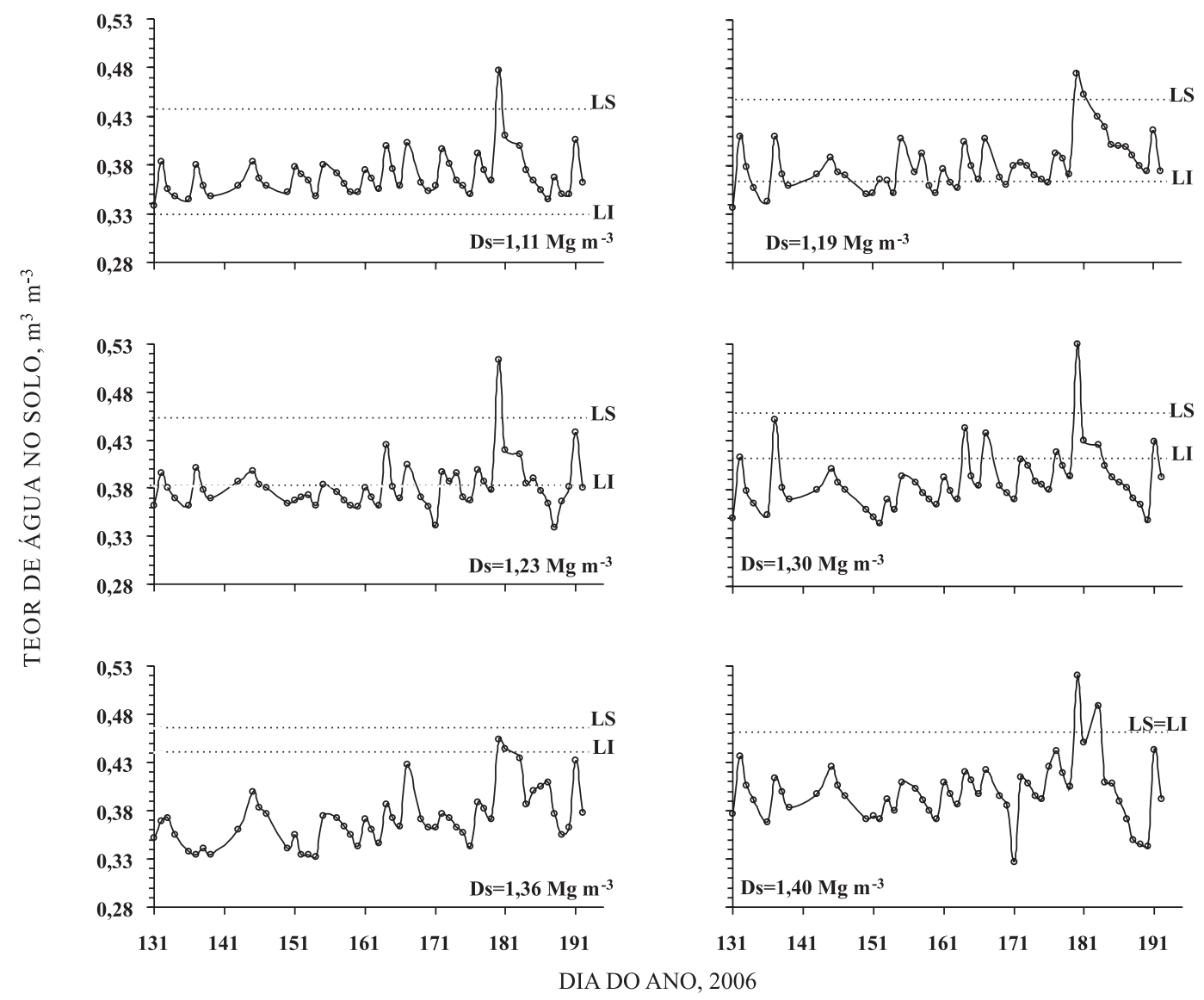

Figura 2. Variação temporal do teor de água no solo em relação aos limites críticos do Intervalo Hídrico Ótimo, Ls - limite superior, e Li - limite inferior do IHO para o período de monitoramento. 


$$
\psi_{\mathrm{RP}}=\left[\left(\frac{\theta_{\mathrm{RP}}}{a \cdot \mathrm{Ds}^{c}}\right)^{\frac{1}{b}}\right]
$$

em que $\Psi_{\mathrm{RP}}$ : potencial de água no solo $(\mathrm{hPa})$ sendo $\mathrm{RP}$ $=2 \mathrm{MPa}$. Assim, o $\Psi_{\mathrm{RP}}$ pode ser utilizado como limite para a aplicação de água no solo antes que a RP limite o crescimento radicular das plantas.

Os resultados indicam que com o aumento da Ds ocorre um aumento do $\Psi$ para $\mathrm{RP}=2,0 \mathrm{MPa}\left(\Psi_{\mathrm{RP}}\right)$; que seja, para a $\mathrm{Ds}=1,11 \mathrm{Mg} \mathrm{m}^{-3}$ pode haver maior secamento do solo em relação à maiores $\mathrm{Ds}$, sem que a RP ultrapasse 2,0 MPa (Figura 3). Entretanto, com o aumento da Ds, a probabilidade do $\Psi<\Psi_{\mathrm{RP}}$ aumenta (Figura 3) expondo a cultura a valores de RP restritiva em condições em que o $\Psi$ não é limitante.

O manejo regionalizado do $\Psi$, embasado na variabilidade espacial da Ds, pode ser utilizado para aplicação diferenciada de água. Nesse caso, o mapeamento da qualidade física do solo e a delimitação de áreas com Ds homogêneas podem ser usados para definir o $\Psi_{\mathrm{RP}}$ para essas áreas e consequentemente para o manejo de água regionalizado, visando ao controle da qualidade física do solo. Dessa forma, por meio do modelo ajustado à curva de retenção de água no solo, pode-se estimar o $\Psi$ em que $\mathrm{RP}=2,0 \mathrm{MPa}$ para as áreas com diferentes Ds (Figura 4).
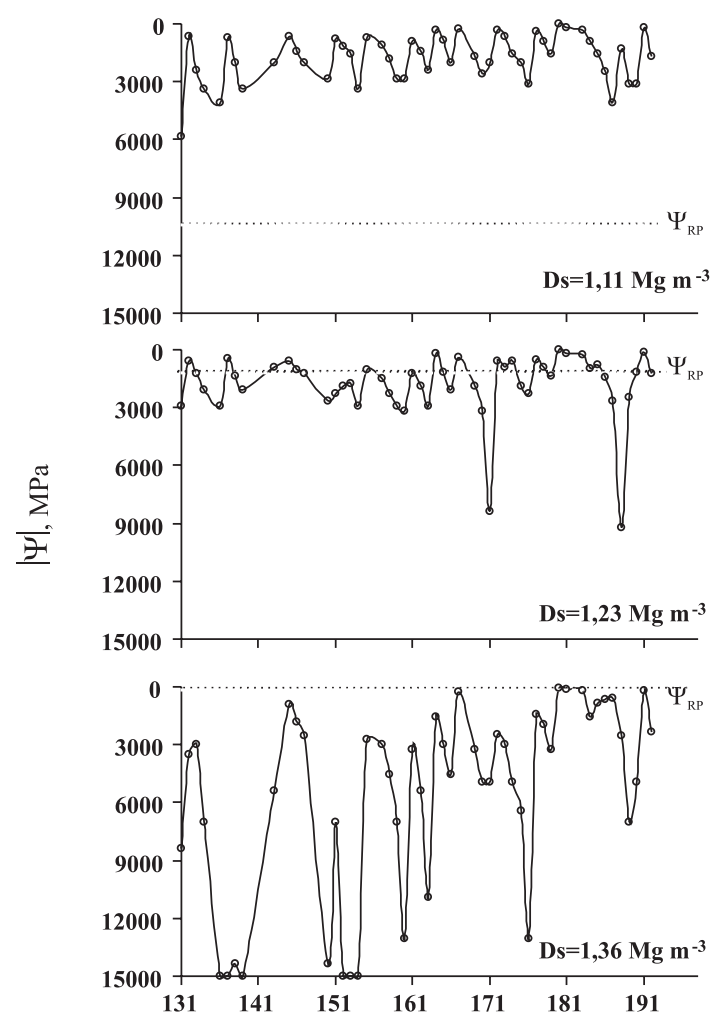

DIA DO ANO, 2006
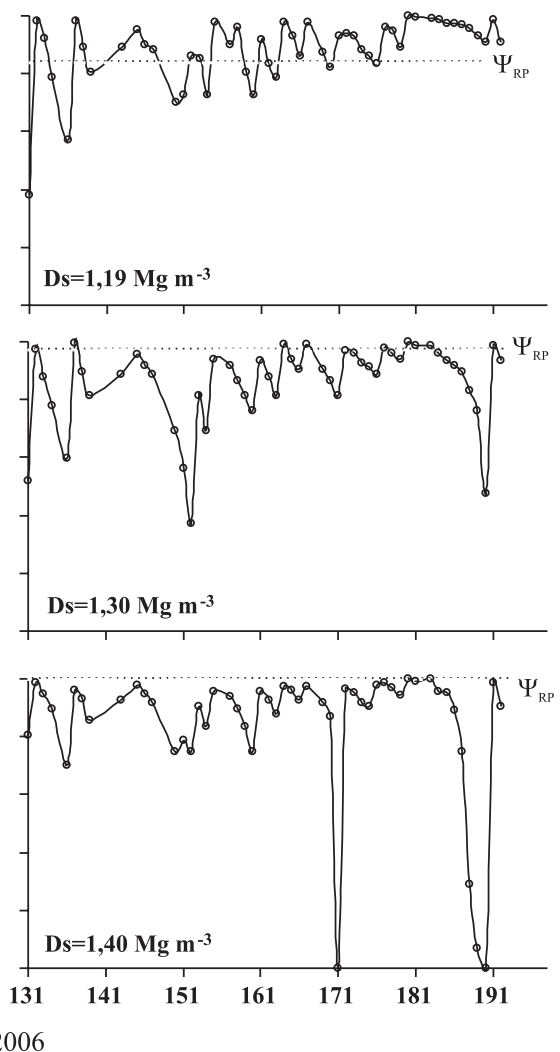

Figura 3. Variação temporal do potencial da água no solo (valores de $\Psi$ em módulo) em relação ao limite crítico de resistência do solo à penetração $\left(\Psi_{\mathrm{RP}}\right)$ para o período de monitoramento. 
e desenvolvimento das plantas, como constatado por Collares et al. (2006).

\section{CONCLUSÕES}

1. O IHO apresentou redução com o aumento da Ds para toda a faixa de valores estudada. O limite superior do IHO foi determinado pelo $\theta_{\mathrm{CC}}$ para Ds $<1,35 \mathrm{Mg} \mathrm{m}^{-3}$. A partir desta Ds, o limite superior do IHO foi determinado pelo $\theta_{\mathrm{PA}}$. O limite inferior do IHO foi determinado por $\theta_{\mathrm{RP}}$ em toda a extensão de Ds. A densidade do solo crítica foi de $1,40 \mathrm{Mg} \mathrm{m}^{-3}$.

2. A utilização do potencial de $-800 \mathrm{hPa}$ como limite para manejo de irrigação expõe a cultura à resistência à penetração restritiva para Ds entre $1,28 \mathrm{e}$ $1,40 \mathrm{Mg} \mathrm{m}^{-3}$.

3. O manejo de água pode ser realizado de maneira diferenciada em áreas com Ds homogêneas visando ao controle da qualidade física do solo para locais com Ds $<$ Dsc.

\section{LITERATURA CITADA}

ARAUJO, M.A.; TORMENA, C.A. \& SILVA, A.P. Propriedades físicas de um Latossolo Vermelho distrófico cultivado e sob mata nativa. R. Bras. Ci. Solo, 28:337-345, 2004.

BENGHOUGH, A.G. \& MULLINS, C.E. Mechanical impedance to root growth: A review of experimental techniques and root growth responses. J. Soil Sci., 41:341-358, 1990.

BENGHOUGH, A.G.; BRANSBY, M.F.; HANS, J.; McKENNA, S.J.; ROBERTS, T. \& VALENTINE, T.A. Root responses to soil physical conditions: Growth dynamics from field to cell. J. Exper. Bot., 57:437-443, 2006.

BEUTLER, A.N.; CENTURION, J.F.; CENTURION, M.A.P.C.; FREDDI, O.S.; SOUSA NETO, E.L.; LEONEL, C.L. \& SILVA, A.P. Traffic soil compaction of an Oxisol related to soybean development and yield. Sci. Agric., 64:608$615,2007$.

BLAINSKI, E.; TORMENA, C.A.; FIDALSKI, J. \& GUIMARAES, R.M.L. Quantificação da degradação física do solo por meio da curva de resistência do solo a penetração. R. Bras. Ci. Solo, 32:975-983, 2008.

BLAKE, G.R. \& HARTGE, K.H. Bulk density. In: KLUTE, A., ed. Methods of soil analysis: Physical and mineralogical methods. 2.ed. Madison, Soil Science Society of America/ American Society of Agronomy, 1986. p.363-375.

BUSSCHER, W.J. Adjustment of flat-tipped penetrometer resistance data to a commom water content. Trans. Am. Soc. Agric. Eng., 33:519-524, 1990.

BUSSCHER, W.J.; BAUER, P.J. \& FREDERICK, J.R. Recompaction of a coastal loamy sand after deep tillage as a function of subsequent cumulative rainfall. Soil Till. Res., 68:49-57, 2002.
BUSSCHER, W.J.; EDWARDS, J.H.; VEPRASKAS, M.J. \& KARLEN, D.L. Residual effects of slit tillage and subsoiling in a hardpan soil. Soil Till. Res., 35:115-123, 1995.

CAMPBELL, G.S. Soil water potential measurement: An overview. Irrig. Sci., 9:265-273, 1988.

CAVALIERI, K.M.; TORMENA, C.A.; VIDIGAL FILHO, P.S.; GONÇALVES, A.C.A. \& COSTA, A.C.S. Efeitos de sistemas de preparo nas propriedades físicas de um Latossolo Vermelho distrófico. R. Bras. Ci. Solo, 30:137$147,2006$.

COLLARES, G.L.; REINERT. D.J.; REICHERT, J.M. \& KAISER, D.K. Qualidade física do solo na produtividade da cultura do feijoeiro num Argissolo. Pesq. Agropec. Bras., 41:1663-1674, 2006.

DANE, J.H. \& HOPMANS, J.W. Pressure plate extractor. In: DANE, J.H. \& TOPP, C., eds. Methods of soil analysis: Physical methods. Madison, Soil Science Society of America/American Society of Agronomy, 2002. Part 4. p.688-690.

EHLERS, W.; KOPKE, V.; HESSE, F. \& BOHM, W. Penetration resistance and root growth of oats in tilled and untilled loess soil. Soil Till. Res., 3:261-75, 1983.

EMPRESA BRASILEIRA DE PESQUISA AGROPECUÁRIA EMBRAPA. Centro Nacional de Pesquisa de Solos. Manual de métodos de análise de solo. 2.ed. Rio de Janeiro, 1997. 212p.

EMPRESA BRASILEIRA DE PESQUISA AGROPECUÁRIA EMBRAPA. Centro Nacional de Pesquisa de Solos. Sistema brasileiro de classificação de solos. 2.ed. Rio de Janeiro, 2006. 306p.

GRABLE, A.R. \& SIEMER, E.G. Effects of bulk density, aggregate size, and soil water suction on oxygen diffusion, redox potential and elongation of corn roots. Soil Sci. Soc. Am. J., 32:180-186, 1968.

IMHOFF, S.; SILVA, A.P.; DIAS JÚNIOR, M.S. \& TORMENA, C.A. Quantificação das pressões crítica para o crescimento das plantas. R. Bras. Ci. Solo, 25:11-18, 2001.

INSTITUTO AGRONÔMICO DO PARANÁ - IAPAR. O feijão no Paraná. Londrina, 1989. 303p. (Circular, 63)

INSTITUTO AGRONÔMICO DO PARANÁ - IAPAR. Cartas climáticas do Paraná: Edição ano 2000, versão 1.0. Londrina, 2000. CD-ROM.

KAY, B.D. \& ANGERS, D.A. Soil structure. In: SUMNER, M.E., ed. Handbook of soil science. Boca Raton, CRC Press, 1999. p.229-276.

KAY, B.D. Rates of changes of soil structure under different cropping systems. Adv. Soil Sci., 12:1-51, 1990.

KAY, B.D.; SILVA, A.P. \& BALDOCK, J.A. Sensitivity of soil structure to changes in organic carbon content: Predictions using pedotransfer functions. Can. J. Soil Sci., 77:655-667, 1997.

KLEIN, V.A. \& CAMARA, R.K. Rendimento da soja e intervalo hídrico ótimo em Latossolo Vermelho sob plantio direto escarificado. R. Bras. Ci. Solo, 31:221-227, 2007. 
KRAMER, P.J. \& BOYER, J.S. Water relations of plants and soils. New York, Academic Press, 1995. 495p.

LEÃO, T.P.; SILVA, A.P.; MACEDO, M.C.M.; IMHOFF, S. \& EUCLIDES, V.P.B. Intervalo hídrico ótimo na avaliação de sistemas de pastejo contínuo e rotacionado. R. Bras. Ci. Solo, 28:415-423, 2004.

LEÃO, T.P.; SILVA, A.P.; PERFECT, E. \& TORMENA, C.A. An algorithm for calculating the least limiting water range of soils. Agron. J., 97:1210-1215, 2005.

LETEY, J. Relationship between soil physical properties and crop production. Adv. Soil Sci., 1:277-294, 1985.

LIBARDI, P.L. Dinâmica da água no solo. São Paulo, EDUSP, 2005. $344 p$.

LOPES, A.S.; PAVANI, L.C.; CORÁ, J.E.; ZANINI, J.R. \& MIRANDA, H.A. Manejo da irrigação (Tensiometria e balanço hídrico climatológico) para a cultura do feijoeiro em sistemas de cultivo direto e convencional. Eng. Agric., 24:89-100, 2004.

REICHARDT, K. Capacidade de campo. R. Bras. Ci. Solo, 12:211-216, 1988.

REICHARDT, K. \& TIMM, L.C. Solo, planta e atmosfera: Conceitos, processos e aplicações. Barueri, Manole, 2004. $477 \mathrm{p}$.

ROMANO, N.; HOPMANS, J.W. \& DANE, J.H. Suction table. In: DANE, J.H. \& TOPP, C., eds. Methods of soil analysis: Physical methods. Madison, Soil Science Society of America/American Society of Agronomy, 2002. Part 4. p.692-698.

ROSS, P.J.; WILLIAMS, J. \& BRISTOW, K.L. Equations for extending water retention curves to dryness. Soil Sci. Soc. Am. J., 55:923-927, 1991.

SAS Institute. SAS/STAT procedure guide for personal computer. 5.ed. Cary, 1999.
SAVAGE, M.J.; RITCHIE, J.T.; BLAND, W.L. \& DUGAS, W.A. Lower limit of soil water availability. Agron. J., 88:844851, 1996

SILVA, A.P \& KAY, B.D. Effect of soil water content variation on the least limiting water range. Soil Sci. Soc. Am. J., 61:884-888, 1997.

SILVA, A.P.; KAY, B.D. \& PERFECT, E. Characterization of the least limiting water range. Soil Sci. Soc. Am. J., 58:1775-1781, 1994.

TOPP, G.C.; GALGANOV, Y.T.; WIRES, K.C. \& CULLEY, J.L.B. Non limiting water range (NLWR): An approach for assessing soil structure. Ottawa, Agriculture and AgriFood Canada, 1994. 36p. (Technical report, 2; Soil Quality Evaluation Program)

TORMENA, C.A.; SILVA, A.P \& LIBARDI, P.L. Soil physical quality of a Brazilian Oxisol under two tillage systems using the least limiting water range. Soil Till. Res., 52:223232,1999

TORMENA, C.A.; ARAUJO, M.A.; FIDALSKI, J. \& COSTA, J.M. Variação temporal do intervalo hídrico ótimo de um Latossolo Vermelho distroférrico sob sistemas de plantio direto. R. Bras. Ci. Solo, 31:211-219, 2007.

TORMENA, C.A.; SILVA, A.P. \& LIBARDI, P.L. Caracterização do intervalo hídrico ótimo de um Latossolo Roxo sob plantio direto. R. Bras. Ci. Solo, 22:573-581, 1998.

VEPRASKAS, M.J. Cone index of loamy sands as influenced by pore size distribution and effective stress. Soil Sci. Soc. Am. J., 48:1220-1225, 1984.

WILLIAMS, S.M. \& WEIL, R.R. Crop cover root channels may alleviate soil compaction effects on soybean crop. Soil Sci. Soc. Am. J., 68:1403-1409, 2004.

ZOU, C.; SANDS, R.; BUCHAN, G. \& HUDSON, I. Least limiting water range: A potential indicator of physical quality of forest soils. Aust. J. Soil Res., 28:947-958, 2000. 
